Rev SINAPSIS, Vol. 8, N 1, Junio 2016

\title{
Solución de Comunicación Telefónica como herramienta de trabajo de los estudiantes del Campus II de la Universidad de Granma, Cuba
}

\section{Solución de Comunicación Telefónica}

Alexander Gorgoso Góngora, Ing. $\left({ }^{1}\right)$

Randy Verdecia Peña, Ing. $\left({ }^{2}\right)$

Guillermo Fonseca Alonso, Ing. $\left({ }^{3}\right)$

$\left({ }^{1}\right)$ Empresa de Telecomunicaciones de Cuba S.A (ETECSA), Granma, Cuba

$\left({ }^{2}\right)$ Empresa de Telecomunicaciones de Cuba S.A (ETECSA), Granma, Cuba

$\left(^{2}\right)$ Empresa de Telecomunicaciones de Cuba S.A (ETECSA), Granma, Cuba

Contacto: alexander.gorgoso@etecsa.cu

Receptado: 15/03/2016 Aceptado: 05/05/2016

\section{Resumen}

La inadecuada comunicación entre los diferentes locales docentes, administrativos y residenciales, Campus II de la Universidad de Granma, motivó la realización de la presente investigación que tuvo como objetivo brindar una solución de comunicación telefónica basada en la interconexión de dos pizarras telefónicas mediante un enlace IP, la cual posibilita una nueva herramienta de trabajo de los universitarios. Para la realización práctica del proyecto se utilizó varios métodos que permitieron la profundización teórica La solución de comunicación consta de 2 nuevas pizarras telefónicas Panasonic KX-TDE 100, las mismas resuelven las necesidades del cliente y se ajustan al presupuesto planificado para dicha inversión. Esta aprovecha la fibra óptica existente en dicha sede y mediante la cual se realiza un enlace punto a punto entre dos optimux 108 para enlazar las pizarras mediante un flujo E1 con la red pública. Además, se realizó un estudio y cálculo de la cantidad de recursos necesarios para la instalación de las pizarras telefónicas. Al término de la investigación se concluyó que con esta solución de comunicación se logra cubrir la solicitud del cliente y permite que las personas que radican en la sede puedan realizar sus llamadas telefónicas de forma eficiente. 
Rev SINAPSIS, Vol. 8, N 1, Junio 2016

Palabras claves: Campus, solución de comunicación, pizarras telefónicas, presupuesto, optimux, fibra óptica, red pública

\title{
Solution of the telephone communication as a tool for working of the students of Campus II of the university of Granma
}

\begin{abstract}
The unproper communication among the different academic, admisnistrative and recidentials rooms, Campus II in the University of Granma, Cuba gave path to make of this research that had as a goal to grant a solution for the communication based on the interconection of two telephone boards, which make posible a new tool of communication. For the practicalof the project It was used several methods that permited to deepen in the theoretical aspects. The solution of the communication is made up of two newly Panasinic boards KX-TDE 100, which solve the needs of the users and they are fitted to the budget for the investment. It takes advantages the optical fiber by means of which it is made a link point to point between optimux 108 to linked the boards thruogh a flow E1 with the public network. It was also made a study calculus of the necessary for installing the telephone boards. It was concluded that with solution of communication it is achieved the to satisfy the application of the users and it permits that the persons of the Campus can make use of the communication tools.
\end{abstract}

Keywords: Campus, communication of solution, telephony board, budget optimux, optical fiber, public network

\section{Introducción}

Las pizarras telefónicas (PBx) han pasado por una transformación rápida a través de los años, las PBx fueron analógicas, pasaron a semi-digitales y luego a totalmente digitales. En la actualidad se están utilizando las llamadas PBx IP que manejan las señales de voz bajo el protocolo de internet, que aportan beneficios para la integración de telefonía informática (CTI). Un PBx IP puede existir como hardware físico, o puede llevar a cabo sus funciones de forma virtual, realizar las actividades de enrutamiento de llamadas de la PBx tradicional o PBx híbrido como un software.(J. Joskowicz, 2013) 
Rev SINAPSIS, Vol. 8, N 1, Junio 2016

Un PBx se refiere al dispositivo que actúa como una ramificación de la red primaria pública de teléfonos, por lo que los usuarios no se comunican directamente al exterior mediante líneas telefónicas convencionales, sino que al estar la PBx directamente conectada a la RTC (red telefónica pública), será esta misma la que enrute la llamada hasta su destino final mediante enlaces unificados de transporte de voz llamados líneas troncales. En otras palabras, los usuarios de una PBx no están asociados con la central de teléfonos pública, ya que es la misma PBx la que actúa como tal, análoga a una central pública que da cobertura a todo un sector mientras que un $\mathrm{PBx}$ lo ofrece generalmente en las instalaciones de una empresa.(ElastixTech, 2016)

Como parte del desarrollo de las comunicaciones en las universidades cubanas, la Empresa de Telecomunicaciones de Cuba (ETECSA) realiza un despliegue de soluciones mediante la instalación de fibra óptica y pizarras telefónicas con el objetivo de lograr una mejora en los sistemas telefónicos en las diferentes sedes de cada provincia.

La Dirección Territorial ETECSA Granma como parte de este proyecto universitario ha desarrollado una serie de acciones que permite dar solución de forma paulatina a algunas sedes que conforman la Universidad de Granma.

Entre las sedes de la Universidad de Granma se encuentra la Sede Universitaria Campus II donde no existe una conexión telefónica que permita una buena comunicación entre sus diferentes locales y además permita satisfacer la demanda solicitada por sus estudiantes.

Una solución de comunicación para la Universidad de Granma del Campus II eliminaría una de las principales problemáticas, garantizando una mejoría en las comunicaciones y satisfaciendo las demandas de sus estudiantes.

El objetivo de este trabajo es confeccionar una solución de comunicación telefónica en la Sede Campus II de la Universidad de Granma, que permita una óptima comunicación entre locales y logre la satisfacción de los estudiantes y personal administrativo.

\section{Materiales y métodos}


Rev SINAPSIS, Vol. 8, N 1, Junio 2016

En la investigación se utilizó métodos teóricos del conocimiento. El de análisis y síntesis permitió valorar y resumir la información disponible acerca de la problemática objeto de estudio, el inductivo-deductivo se empleó para establecer generalizaciones sobre la base del análisis de las teorías, tendencias y criterios de profesionales que han aplicado estas tecnologías; además del razonamiento e integración de la información obtenida en el proceso de investigación y arribar a propuesta de comunicación. Finalmente, la abstracción y concreción se utilizó para reflejar y reproducir las cualidades, regularidades generales, estables y necesarias de la propuesta.

Para crear la solución de comunicación telefónica se partió de la solicitud realizada por la rectora de la Universidad de Granma y del presupuesto con que contaba para la obtención del equipamiento. Además, se aprovechó la fibra óptica de ETECSA existente hasta la Sede Universitaria con el objetivo de conectar a flujo la pizarra telefónica.

Con estos datos se procedió a realizar la solución de comunicación con la instalación de dos pizarras telefónicas KX-TDE 100, 2 optimux 108, el empleo de la fibra óptica que llega a la sede y el listado de extensiones para determinar los materiales necesarios para la instalación.

\section{Resultados}

El Campus II es una sección de la universidad de nueva creación, las misma se encuentra con un bajo nivel de comunicación telefónica para la cantidad de estudiantes y personal administrativo. Por esta razón la máxima dirección de la Universidad de Granma emite a la Empresa de Telecomunicaciones de Cuba la siguiente carta: 


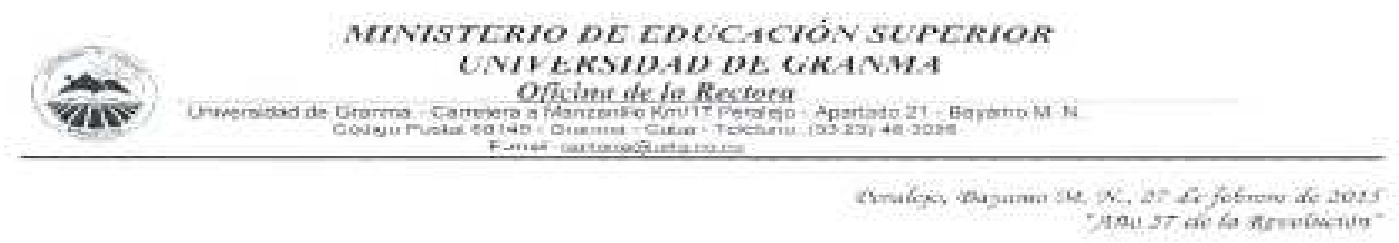

A. Oncina Empresa Dereccion Tenritoriai-Granma ETECSA.

Ret: Solcitud de venta de pizarra teletbrica con capacidad pera 170 Exiensiones

Estimarsos coimpañove

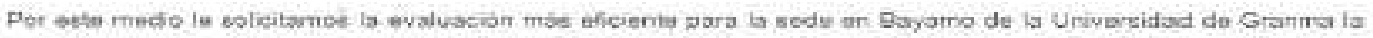

cual se encuentra en proceso de fecminación de 54 inversion $\mathrm{v}$ demanda una pizacra teleforica para 170

exteristunes

\begin{tabular}{|c|c|c|c|}
\hline \multirow{2}{*}{ Localue: } & \multicolumn{2}{|c|}{ Tipo Exterosisn } & \multirow{2}{*}{ Cantidad } \\
\hline & Anaiogico & Muthunaa & \\
\hline Eocio Adfministrativo & 2 & 1 & 10. \\
\hline Oentiles Actoves Friucipal. & & 9 & 1 \\
\hline Dormitorio 2 & 23 & & 23 \\
\hline Dormitorio 1 & 48 & & 48 \\
\hline Qormitorio 3 & 48 & & 48 \\
\hline Laboratocios Docentes & 8. & 1 & 9 \\
\hline Aulas y Otros & 6 & 1 & 6. \\
\hline Diblioteca & 1 & & 1. \\
\hline Lotial de Profesores & 1 & & 1 \\
\hline Lepartamentos Locentes 1 & 3 & 1 & is \\
\hline $\begin{array}{l}\text { Cocina Comedor } \\
\text { Almacèn }\end{array}$ & 2 & & $\begin{array}{l}2 \\
2\end{array}$ \\
\hline Servicio Benerales & 1 & 1 & 2 \\
\hline Mantanimiento & \pm & & $t$ \\
\hline Dopertamontos Dosontos 2 & 口 & $i$ & S \\
\hline Total & & & 109 \\
\hline
\end{tabular}

Anexo: Uticacion por objetos y locales de las extensiones:

Soliving nomtialos
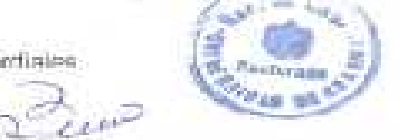

ing. M. Sc. Narcy Bueno Figueras, Prof. Auxilias

Rectora

Documento de solicitud del cliente

Después de analizar dicho documento se procedió a evaluar que pizarras podrían cumplir las expectativas del cliente valorando la capacidad y el presupuesto con que contaba el cliente. La solución ideal es mediante una pizarra telefónica KX-TDE 600 que pueda resolver el problema inmediato y futuro, pero por la demora en que se podría adquirir el equipamiento por ETECSA, la solución se propone mediante la instalación de 2 pizarras telefónicas KX-TDE 100.

Con esas dos pizarras se puede cubrir 136 extensiones analógicas, 8 extensiones digitales y 5 IP; las mismas no se cubre la demanda, pero el costo de la propuesta tiene un valor aproximado a lo planificado en su presupuesto de inversión.

En la figura 1 se muestra la topología general de la solución donde se enlazarán las pizarras mediante enlaces IP. Estas dos pizarras se ubicarán una al lado de la otra. El local seleccionado para el montaje de las mismas es el nodo del campus en cuestión ya 


\section{Rev SINAPSIS, Vol. 8, N 1, Junio 2016}

que cumple con las condiciones de aterramiento y clima siguiendo las normas para la instalación de pizarras telefónicas. Una de estas pizarras se conectará a la red pública mediante un flujo E1, esto se logra mediante un enlace punto a punto entre dos optimux 108, dispositivos de transmisión que garantizan los $2 \mathrm{Mbps}$ para el enlace, el medio físico que se utilizará es la fibra óptica de ETECSA que está en el mismo nodo de la sede universitaria. La salida hacia la red pública de la otra pizarra se enrutará por el enlace IP existente con la otra PBx para poder salir mediante el flujo E1.

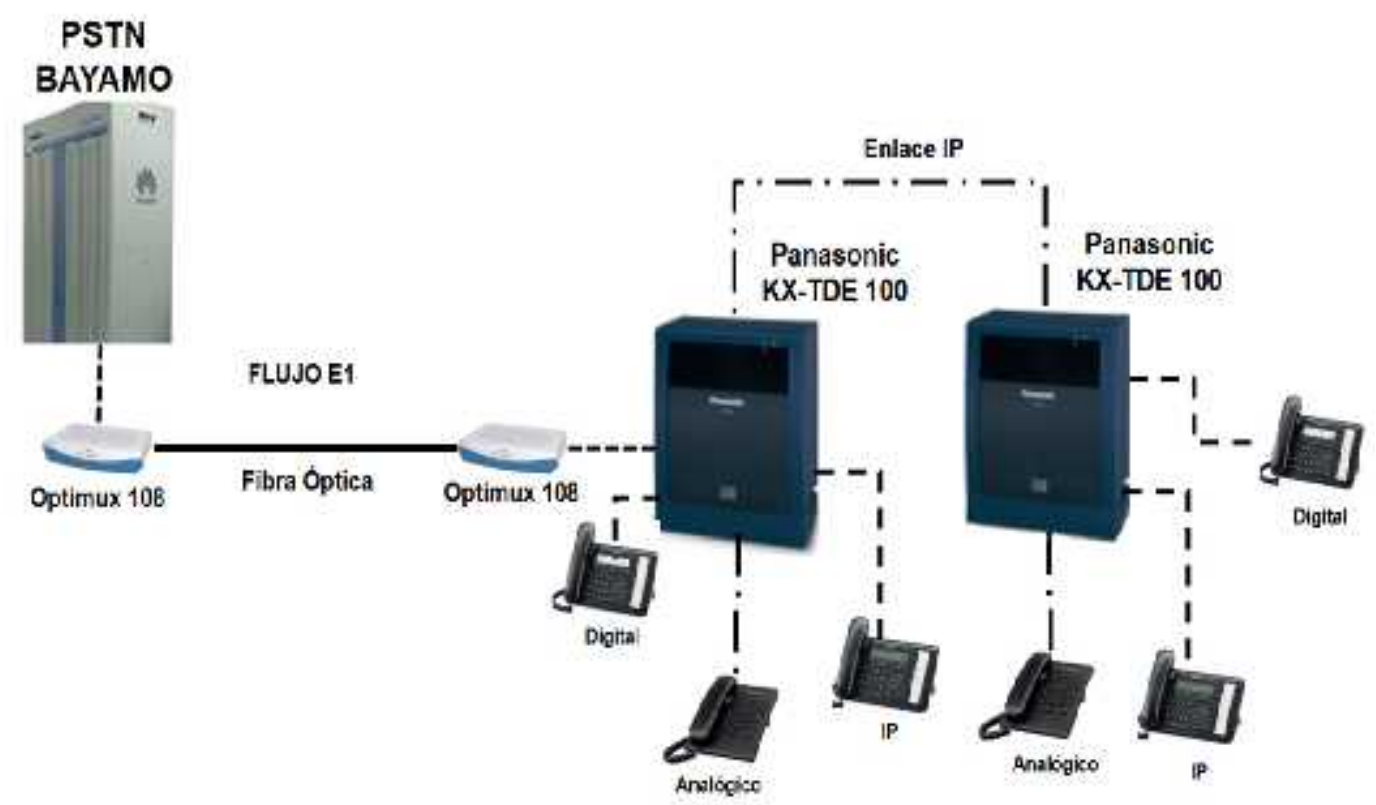

Figura $\mathrm{N}^{0} 1$ Topología general de la solución

La figura 2 refleja el arco de numeración a utilizar, en este caso son dos centenas

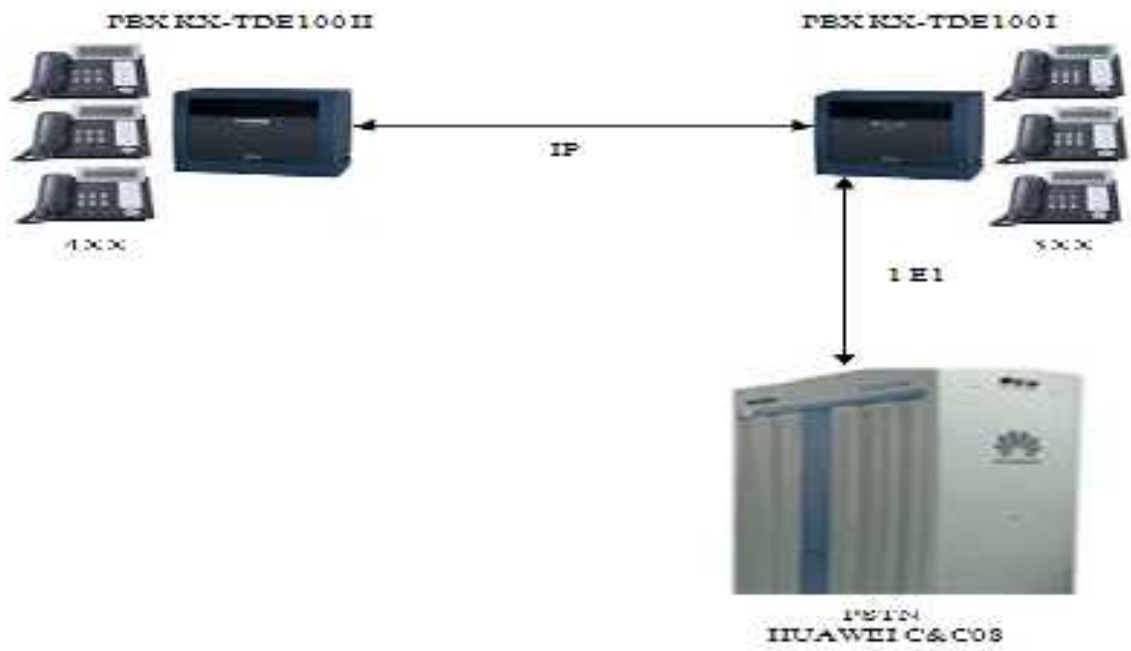

Figura $\mathrm{N}^{0} 2$ Configuración de las pizarras

Solución de Comunicación Telefónica 


\section{Rev SINAPSIS, Vol. 8, N 1, Junio 2016}

Las pizarras Panasonic KX-TDE100 se configuran de acuerdo a los requerimientos del usuario, que define los servicios que desea implementar, teniendo en cuenta las facilidades y bondades que brinda la misma. Una vez teniendo esos datos se procede a la configuración de los arcos de numeración pública asignados; la forma de marcación, tanto interna como externa, así como precisa la manera en que se manejarán las llamadas en entrada y en salida a través del flujo E1, entre otras especificaciones. Para una mejor comprensión detallaremos los elementos de programación más importantes:

La PBX 1 presenta una capacidad máxima de 72 puertos de extensiones analógicas y 8 puertos de extensiones digitales, mientras que la PBX 2 tiene instalados 64 puertos analógicos.

Se asignaron dos centenas de numeración pública, desde la 419300 a la 419499, y como la capacidad en cada una de las pizarras lo permite, decidimos asignarles a las extensiones de la PBX 1 el arco de numeración público 4193XX y a la PBX 2 el arco de numeración público 4194XX, la numeración de las extensiones se conformó con los últimos tres dígitos de dicha numeración, por lo que son $3 \mathrm{XX}$ y $4 \mathrm{XX}$, como se muestra 3 y 4 respectivamente

\begin{tabular}{|c|c|c|c|c|c|c|c|c|c|c|c|c|}
\hline \multirow{2}{*}{ Uan } & \multicolumn{2}{|c|}{ 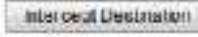 } & \multicolumn{2}{|c|}{ rinceps Nu Ni swer ims } & \multicolumn{2}{|c|}{ DUK CLP } & \multirow{2}{*}{$\begin{array}{l}\text { Oofenit } \\
\text { Pert Tyms }\end{array}$} & 09.012 & Ovivil? & Opiont & Opien 2 & \multirow[t]{2}{*}{ 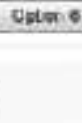 } \\
\hline & tin & $\begin{array}{l}\text { Furasikin } \\
\text { Wutituat }\end{array}$ & 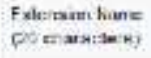 & sheit: & sint & $\mathrm{mnt}$ & & $\begin{array}{l}\pi: x \\
7 n\end{array}$ & thene & Waner ame ip & $\cos$ & \\
\hline \multirow[t]{10}{*}{5} & 1 & 300 & & Thyzlcos: & 1 & , & S-Hvord & & (4) & 1 & 54 & \\
\hline & 2 & 3ni & & Phy:iad & 1 & 2 & Soltylural & $21 \pi$ & & 1 & $t$ & \\
\hline & 3 & Juz & & Pryesal & 1 & J & sitybre & all & & 1 & 1 & \\
\hline & 4 & 303 & & Fhystcal & 4 & 4 & Silyord & GLT & & 1 & t & \\
\hline & : & 304 & & Phyalcal & 1 & 5 & S-tyora & & (twiv) & 1 & 2 & \\
\hline & 6 & 305 & & Alyoxial & 1 & A & S. Hyarral & 81 1 & & 1 & 1 & \\
\hline & 7 & JUE & & thyesat & 1 & 7 & stiblons & 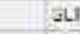 & & t & 1 & \\
\hline & $x$ & 207 & & Fhysi:al : & 1 & 0 & Stlybrd & 3LT & & 1 & 1 & \\
\hline & 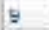 & $30 \mathrm{~ns}$ & & Thvar:al & 1 & xne1 & S-HynralsilT & $=1 T$ & & 1 & 1 & \\
\hline & 10 & $3 n \theta$ & & Dly $y=1$ & 1 & snes & 8. HybrassiT) & R1T & & $t$ & $t$ & \\
\hline \multirow[t]{10}{*}{ रु } & 11 & 310 & & Phosecal & 1 & XUF. & if Hybnd(6L) & 24 & & 1 & 1 & \\
\hline & t. & $2 t$ & & Fhysical & 1 & $x 004$ & S-lyond(sit) & ZLT & & 1 & 1 & \\
\hline & 11. & 312 & & Throlene & 1 & mnst & Setwhrasit) & $\Rightarrow 1 T$ & & 1 & 1 & \\
\hline & 14 & s13 & & Flyosical & 1 & ShaA & 8 Hydrafsit) & $81 \mathrm{~T}$ & & $t$ & 1 & \\
\hline & $1=$ & j14 & & thoseal & 1 & $x \in=1$ & Gitybra(sul) & BLI & & 1 & 1 & \\
\hline & 10 & 315 & & Fhysica & 1 & $x 0 p 0$ & ShlvordsLTh & ILT & & $t$ & 1 & \\
\hline & 11 & 346 & & Thuarad & 1 & nxnPy & S-Hyhra(S-DOPT & Na & innecter & 1 & 1 & \\
\hline & 13 & $31 y$ & & Hryariat & 1 & axusp? & X Hyturass:LWT & Sat & inmede & $t$ & t & \\
\hline & 17. & วน & & Physcal & 1 & DXUP & \&Iybra(s UPI & Not & sonsterer & $t$ & 1 & \\
\hline & 20 & 219 & & Fhystal & 1 & DODPA & S-lybrds-Det & Ne & Vonsctoe & 1 & 1 & \\
\hline \multirow[t]{2}{*}{$\$$} & 21 & 320 & & Mrratal & 1 & monns & S-Hyle dFONMT & & Sine isfar & $t$ & 1 & \\
\hline & 2 & ख्या & & -hyous & 1 & Inonym & S Hyturass INT & sut & Eumuta: & 1 & t & \\
\hline
\end{tabular}

Figura $\mathrm{N}^{0} 3$ Arco de numeración PBx 1 
Rev SINAPSIS, Vol. 8, N $^{\circ}$ 1, Junio 2016

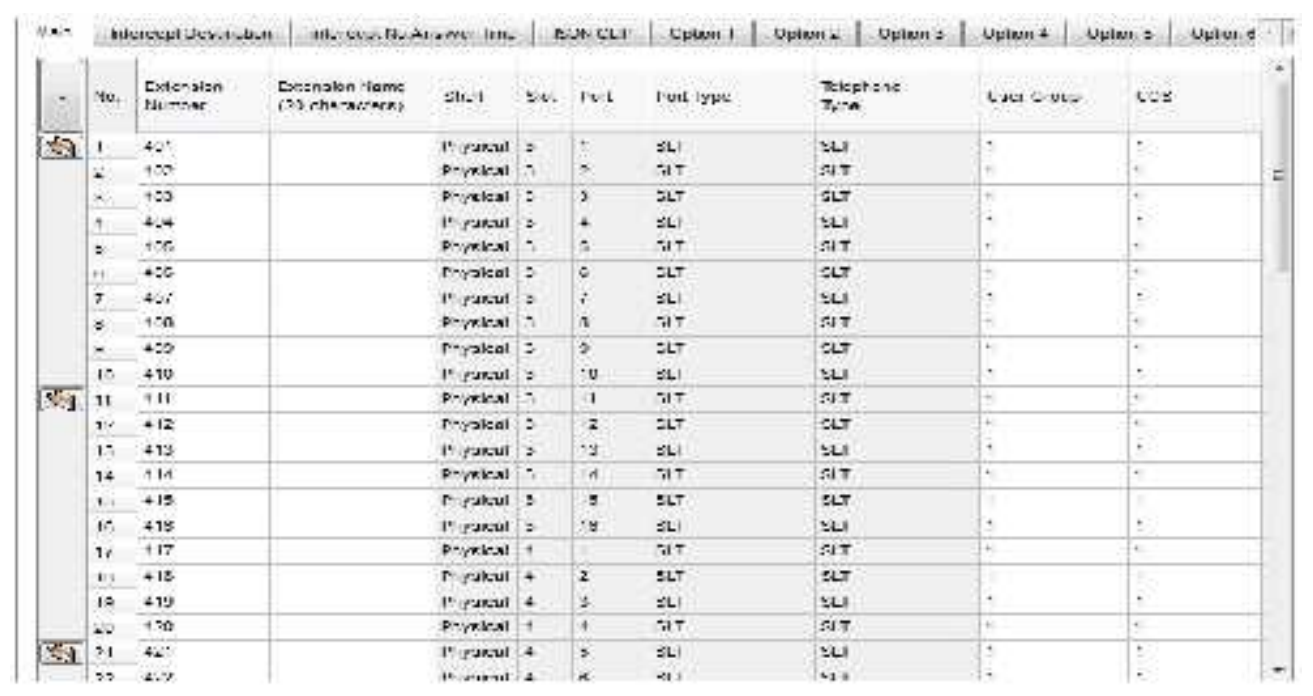

Figura $\mathrm{N}^{0} 4$ Arco de numeración PBx 2

En la institución solamente se habilitó un puesto de operadora, independientemente que hay dos pizarras. Cuando se marca el código de acceso a operadora desde cualquier extensión del sistema, la llamada se direcciona a la extensión 300 que es la habilitada como operadora ilustrada en la figura 7.

El enlace privado se realizó a través de canales de enlace IP. En la figura siguiente se muestra el gabinete virtual de la PBX.

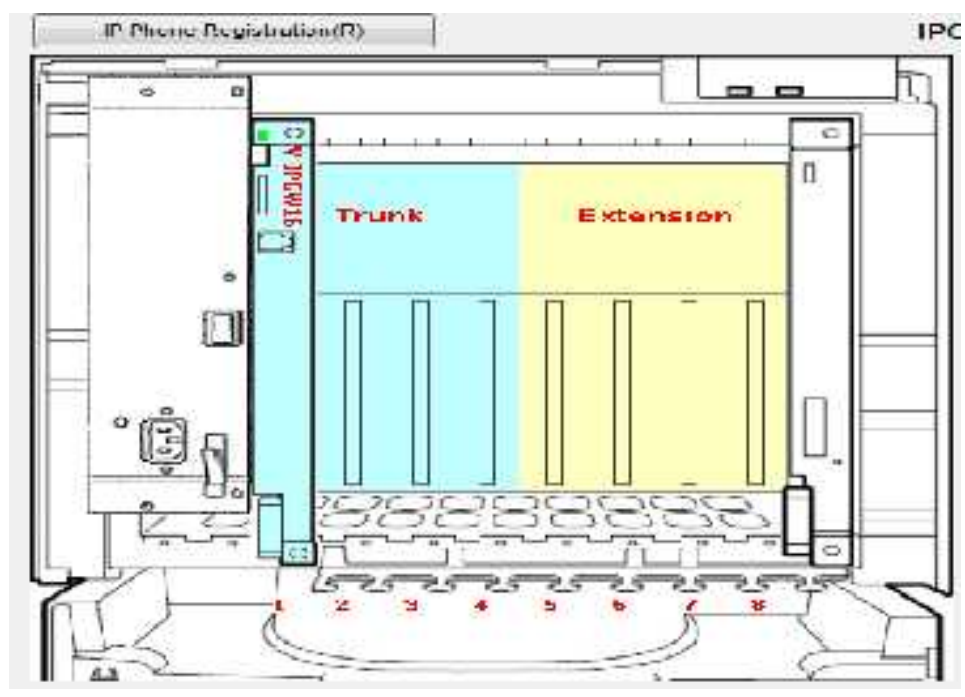

Figura $\mathrm{N}^{0} 5$ Gabinete virtual PBx 1

Para realizar este tipo de enlace se instaló, en ambas pizarras, una tarjeta hija sobre la IPCMPR denominada DSP64, que nos provee de 64 procesadores de voz y nos habilita 
Rev SINAPSIS, Vol. 8, No 1, Junio 2016

a cada PBx como un Gateway para la comunicación entre el mundo IP con el mundo TDM tanto interna como externa.

Al instalar la DSP64 se puede configurar entonces el gabinete virtual con las tarjetas necesarias para poder realizar el enlace troncal IP entre ambas pizarras. Específicamente en este caso se utilizó la tarjeta virtual V-IPGW16 que nos proporcionará 16 canales H323 para el enlace.

\begin{tabular}{|c|c|c|c|c|}
\hline \multicolumn{2}{|c|}{ fiK SeThgs $(K)$} & \multicolumn{2}{|c|}{ GW Set Th gs( } & \multirow[t]{2}{*}{ It ni Patern(H) } \\
\hline Mati & nurgnrg f.al & In ynrg $c . a$ I & TIrfer & \\
\hline \multicolumn{4}{|c|}{ [esa plivil } & Walue \\
\hline \multicolumn{4}{|c|}{ Oa:etesper Avalabe } & Disable \\
\hline \multicolumn{4}{|c|}{ [al signeiling Mrde } & Dute: \\
\hline \multicolumn{4}{|c|}{ Co:ckczpor Connzetcn Chscling hterral ( $\left.{ }^{2} 303\right)$} & 2 \\
\hline \multicolumn{4}{|c|}{ Frimary Gatelee 7 er F Ardiese } & 192.168 .1 .3 \\
\hline \multicolumn{4}{|c|}{ Frimary Gatek:e zer Por. Number } & $1 / 7 y$ \\
\hline \multicolumn{4}{|c|}{ Sevandary Galeszenel IP Addi ass } & 192.160 .1 .4 \\
\hline \multicolumn{4}{|c|}{ Seconoary t3ateceeper Hort $\mathrm{H}$ smber } & 1719 \\
\hline \multicolumn{4}{|c|}{ 1.225 Port Nunser } & 1720 \\
\hline \multicolumn{4}{|c|}{ Has Hert number } & 1719 \\
\hline \multicolumn{4}{|c|}{ QSIC Cornestiorlsas Turne ing TCR $\Gamma$ crt $N$ umbsr } & 1718 \\
\hline \multicolumn{4}{|c|}{ 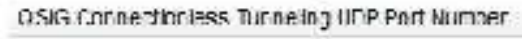 } & 1717 \\
\hline \multicolumn{4}{|c|}{ F.323 Dynanc Tert Humber } & 10000 \\
\hline \multicolumn{4}{|c|}{ FTCF Pachet 3tudi y Aullity } & Cneble \\
\hline \multicolumn{4}{|c|}{ FTCF Packet rtarval } & 25 \\
\hline \multicolumn{4}{|c|}{ FTP Q03 Available } & $T_{0} \mathrm{\Xi}$ \\
\hline \multicolumn{4}{|c|}{ 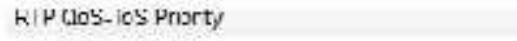 } & 7 \\
\hline \multicolumn{4}{|c|}{ RTP QOS-TOS TYPE } & Nernal \\
\hline \multicolumn{4}{|c|}{ RTP DNS-DFC.P } & \\
\hline
\end{tabular}

Figura $\mathrm{N}^{0} 6$ Configuración de la tarjeta DSP

En el caso de la PBX 2 utilizará este enlace para:

- Realizar llamadas a las extensiones de la PBX 1 de forma transparente.

- Llamadas a la operadora.

- Llamadas hacia la red pública haciendo tránsito a través de la PBX 1 y saliendo por el flujo E1 conectada a la misma, mostrado en la figura 7. 
Rev SINAPSIS, Vol. 8, No 1, Junio 2016

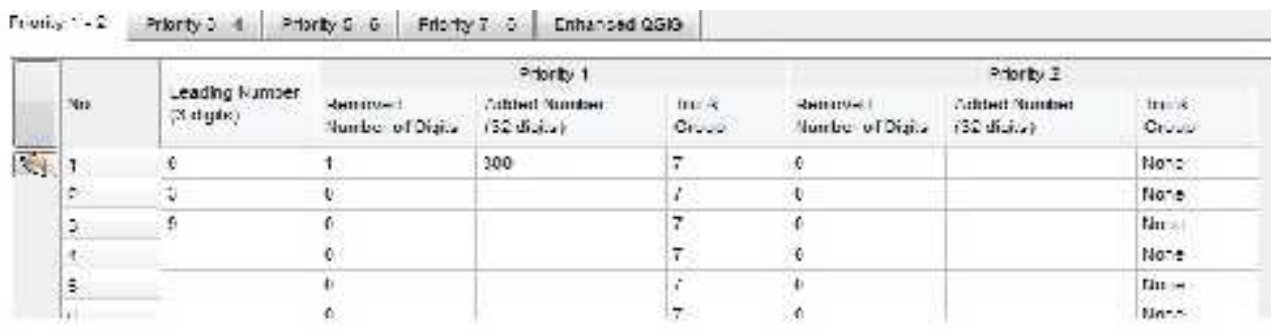

Figura $\mathrm{N}^{0} 7$ Configuración PBx 2 para la salida hacia PSTN

En el caso de la PBX 1 utilizará este enlace para:

- Realizar llamadas a las extensiones de la PBX 2 de forma transparente.

- Enrutar el tránsito proveniente de la red pública y que tienen como destino las extensiones 400 de la PBX 2 como se refleja en figura 8.

\begin{tabular}{||l|l|l|l|l|}
\hline Prinnity 1-7 & Priority 3-4 & Priority 5-6 & Priority 7-8 & Enhanced 0SIG \\
\hline
\end{tabular}

Figura $\mathrm{N}^{0} 8$ Configuración PBx 1 para la salida hacia PSTN

Para realizar el enlace del sistema con la red pública, en este caso con la central Huawei C\&C08, se utilizó un flujo de 30 canales E1.

Este enlace se habilitó de acuerdo a los requerimientos que necesita. Los principales elementos que se deben tener en cuenta son el tipo de señalización en este caso R2, la numeración que transitará por el flujo, tanto en entrada como en salida, así como la cantidad de dígitos, forma de realizar la facturación, dirección de los canales, en este caso todos son en entrada y salida, etc.

Cuando el usuario externo marca la numeración de cualquiera de los arcos definidos, la Central Pública solamente enviará a través del flujo E1 las últimas tres cifras, siendo la PBx 1 la encargada de definir el destino de esa numeración.

Existen dos destinos posibles: 
- Si la numeración coincide con la de las extensiones internas de la PBx 1 entonces se define el timbre en las mismas, ver figura 10.

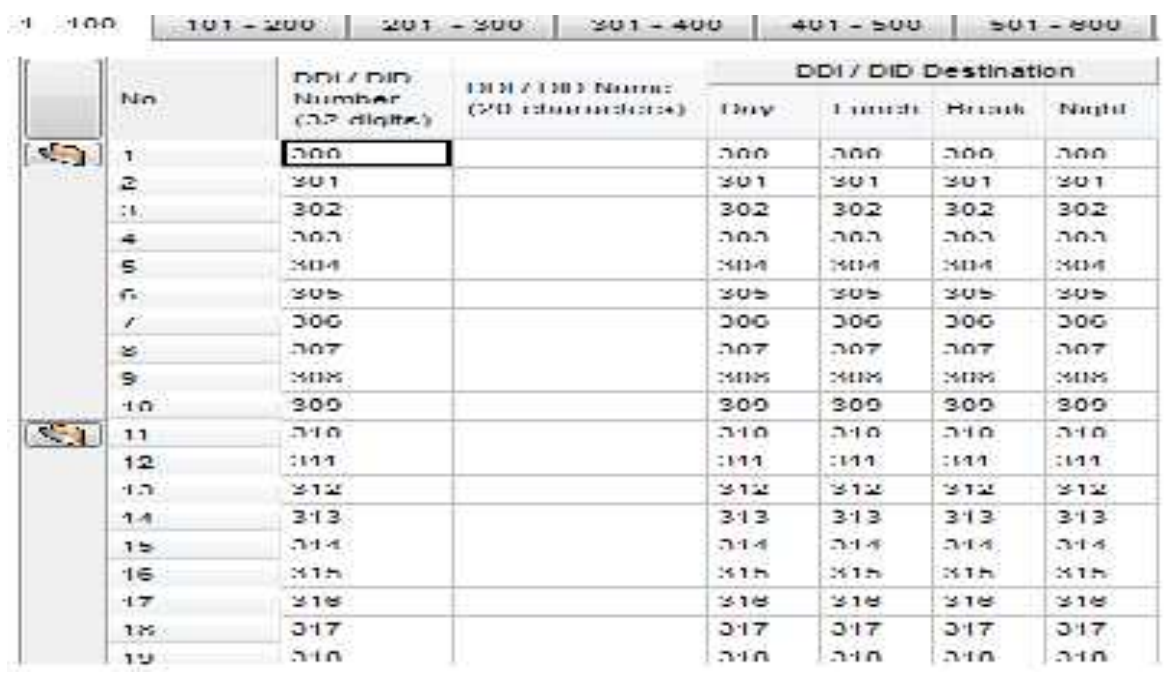

Figura $\mathrm{N}^{0} 9$ Enrutamiento de la llamada

- Si la numeración no coincide con la de las extensiones internas de la PBX 1 pero está definida en la figura 9, entonces la reenruta a través del enlace privado hacia la PBX 2.

En la PBx 1 las extensiones marcan el 9 para acceder a la red pública. De acuerdo a la figura 9 cuando la pizarra reconoce el dígito de acceso a los troncales enruta la llamada a través del flujo E1 hacia la PSTN.

En la PBx 2 las extensiones marcan también el 9 para acceder a la red pública y de acuerdo a la figura 7 la llamada se enruta a través del enlace privado hacia la PBx 1, esta a su vez realiza el análisis de la numeración que recibe y al detectar que no es parte de su numeración interna entonces se rige por la figura 8 y la envía hacia la PSTN a través del flujo E1.

Para la instalación de estas pizarras telefónicas se hace necesaria la realización de un levantamiento de los materiales que se utilizarán para la puesta en marcha de las misma. A continuación, se muestra el listado de los materiales necesarios: 
Rev SINAPSIS, Vol. 8, N 1, Junio 2016

\begin{tabular}{|c|c|c|}
\hline Descripción & Unidad & Cantidad \\
\hline GRAPA DE 2 PATAS $1 / 2 " 13 \mathrm{MM}$ & $\mathrm{U}$ & 100 \\
\hline MODULO EMPATE N/PRESURIZ 550/43/8-300 & $\mathrm{U}$ & 2 \\
\hline PICABON VERDE SECO P/ RED SOTERRADA & $\mathrm{U}$ & 1 \\
\hline PRESILLA "S" SUJECION ALAMBRE TELEFONICO & $\mathrm{U}$ & 50 \\
\hline GRAPA PLAST. C/CLAVO 1" DIA.4MM (1*100) & $\mathrm{U}$ & 15 \\
\hline CONECTOR DE ENTRADA CON GEL/LOGO ETECSA & $\mathrm{U}$ & 10 \\
\hline CAJA CONECTORA RJ11 2 VIAS C/GEL BEIGE & $\mathrm{U}$ & 65 \\
\hline CAJA CONECTORA RJ11 4 VIAS C/GEL BEIGE & $\mathrm{U}$ & 9 \\
\hline MONTAJE INTERIOR 2 VIAS (CII 2X1X0.5) & M & 1500 \\
\hline CABLE TELEFONICO CL 50X2X0.4 & M & 134 \\
\hline SOPORTE P/10REGLETA 10P R27022-20 & $\mathrm{U}$ & 3 \\
\hline TOMACORRIENTE DOBLE 15A 125V & $\mathrm{U}$ & 2 \\
\hline TAPA P/TOMACORRIENTE DOBLE 15A & $\mathrm{U}$ & 2 \\
\hline CAJA MECAN 4X2 P/CANALETA ELECTROCANALI & $\mathrm{U}$ & 2 \\
\hline CABLE ELECT CLASE5 12AWG 750V VERDE-AMA & M & 40 \\
\hline BRIDA USO EXTERIOR 7,6X368MM ANXLARG & $\mathrm{U}$ & 200 \\
\hline TAPE FRICCION 2X0.15X60 & $\mathrm{U}$ & 1 \\
\hline TAPE DE GOMA 2" & $\mathrm{U}$ & 1 \\
\hline CAJAS ESTANCO IP-55 LEGRAND 220X170MM & $\mathrm{U}$ & 1 \\
\hline CAJAS ESTANCO IP-55 LEGRAND 155X110MM & $\mathrm{U}$ & 4 \\
\hline ALAMBRE BAJANTE TELEFONICO 2X2X1 & M & 600 \\
\hline CLAVO P/CONCRETO 2" & KG & 2 \\
\hline TORNILLO OJO RECTO 5/8 X 10 & $\mathrm{U}$ & 8 \\
\hline CABLE TELEFONICO CL 10X2X0.4 & M & 140 \\
\hline CABLE TELEFONICO CLA 50X2X0.4 & $\mathrm{M}$ & 150 \\
\hline CABLE TELEFONICO CLA 20X2X0.4 & M & 510 \\
\hline MONTAJE INTERIOR 2 VIAS (CII 2X1X0.5) & M & 300 \\
\hline TAPE PVC 3/4X33 NEGRO/ROJO/VERDE/AMARILL & M & 3 \\
\hline MORDAZA PLASTICA P/BAJANTE EN SOPORTE L & $\mathrm{U}$ & 50 \\
\hline PANEL METALICO CON PUERTA 600X500X250MM & $\mathrm{U}$ & 1 \\
\hline CAJA ELECT POLICARBONATO IP55 250X250X90 & $\mathrm{U}$ & 6 \\
\hline
\end{tabular}


Rev SINAPSIS, Vol. 8, No 1, Junio 2016

\begin{tabular}{|l|c|c|}
\hline MODULO EMPATE VERTICAL EMYCO 100-200 P & $\mathrm{U}$ & 2 \\
\hline CAJA TERMINAL 20/21 PARES INSERC C/COLA & $\mathrm{U}$ & 2 \\
\hline HERRAJE/SOPORTE/BLOQUE 50P R27230-50-05 & $\mathrm{U}$ & 8 \\
\hline REGLETA D/DESCONEXION 10PARES R2700220-1 & $\mathrm{U}$ & 34 \\
\hline
\end{tabular}

\section{Discusión}

Las pizarras privadas IP han tenido gran aceptación en todos los sectores de nuestro país. Con las PBx IP logra trabajar fuera de la oficina mediante los sistemas inalámbricos implementados, se fortalece las capacidades de equipamiento, proporciona una solución unificada para que las empresas satisfagan sus necesidades de comunicación telefónica en uno o varios sitios, tanto en el presente como en el futuro. Además, permite implementar una solución de correo de forma centralizada y personalizada en múltiples idiomas y la eficiente administración de las llamadas. (Panasonic, 2011)

Para llegar a la solución de comunicación se realizó un estudio del equipamiento que comercializa la Empresa de Telecomunicaciones de Cuba a todos los sectores del país, así como valorar el equipamiento y presupuesto del cliente; para poder en un futuro unificar todos los servicios. Después de realizar todo un análisis de las cuestiones anteriormente reflejadas la tecnología de pizarras telefónicas a utilizar es la Panasonic por toda la gama de servicio como se exponen en (Panasonic España, 2012).

La estructura de comunicación está compuesta por dos pizarras KX-TDE 100 de Panasonic que responden a las necesidades del cliente. Además, se enlazan por vía IP mediante las tarjetas DSP colocadas en ambas pizarras. Este enlace permite que las llamadas entre las dos pizarras sean a tres dígitos. Además, permite que las extensiones de la pizarra 2 puedan utilizar el flujo E1 instalado en la pizarra 1 como enlace con la central telefónica.(Panasonic, 2010b)

El acceso a red públicas se realizará mediante un flujo E1 que es un canal de voz, datos y video, con un ancho de banda de $2.048 \mathrm{Mbps}$, en los que se multiplexan hasta 30 canales de información en unos o dos pares de cobre o 2 hilos de fibra óptica con se informa (Panasonic, 2009). Con la instalación de los 30 canales de voz en la pizarra 1 cada extensión podrá tener un número asociado de los arcos de numeración. 
Para lograr obtener un enlace punto a punto a 2 Mbps entre la PBx y la central telefónica se utilizó un equipo de transmisión. Este optimux 108 tiene varias interfaces de salida E1, T1y fast ethernet y soporta interfaces de fibra como simple modo, multimodo, etc.(Communications, 2010)

El enlace físico es un aspecto muy importante para brindar al cliente una eficiente estructura de comunicación. En la propuesta de comunicación se reutiliza la fibra óptica existente en la sede de la universitaria. Con este medio físico se alcanzarán los mejores valores para lograr una buena comunicación telefónica. (Velásquez, 2012)

Después de realizado el diseño de la solución de comunicación se realizó la configuración de las extensiones de cada pizarra con las limitaciones especificada por el usuario. Además, se determinaron los parámetros en la tarjeta de flujo de la pizarra que se enlazará con la central telefónica, así como la configuración del enlace privado entre las dos PBx. (Panasonic, 2010a)

\section{Conclusiones}

La solución de comunicación de la Universidad de Granma, Campus II mediante dos pizarras telefónicas Panasonic KX-TDE 100, las cuales se enlazarán entre ellas por vía IP y con la centra pública mediante un enlace E1, constituye una solución de que permite el establecimiento de las llamadas entre los diferentes locales y facilita el logro de los objetivos de dicho centro estudiantil universitario.

\section{Bibliografía}

1. Communications, D. (2010). Optimux-108 , Optimux-106. Retrieved from http://www.bestdatasource.com/rad/data_sheets/OP-108.pdf

2. ElastixTech. (2016). -CenPBXrtal TeelBfonica - Funcionamiento de la PX. Telefonía IP con Asterix. Retrieved from http://elastixtech.com/fundamentos-detelefonia/pbx-central-telefonica/

3. Joskowicz, J. (2013). Conceptos de telefonia corporativa. Instituto de Ingeniería Eléctrica Universidad de Montevideo, URUGUAY, 10, 1-76. Retrieved from http://www.fing.edu.uy/iie/ense/asign/ccu/material/docs/Conceptos de Telefonia Corporativa.pdf

4. Panasonic. (2011). Sistema de Comunicación para Oficina. Panasonic Ideas for Life, 1105. Retrieved from 
Rev SINAPSIS, Vol. 8, № 1, Junio 2016

http://cast.mx/Panasonic/Panasonic_Sistemas_de_Comunicacion_para_Oficina_Ca talogo_General_PBX_general_catalogo_2011.pdf

5. Panasonic España, S. (2012). Formación Nivel Experto Formación Niv. Panasonic Ideas for Life. Retrieved from http://imatel.es/manuales/TDE.pdf

6. Panasonic, S. (2009). IP-PBX Híbrida Manual E LACE DIGITAL E1. In Manual E1 (pp. 11-20).

7. Panasonic, S. (2010a). Manual de instalación Central pura IP KX-TDE100.

8. Panasonic, S. (2010b). Primeros pasos para tarjetas IP virtuales Central pura IP KXTDE100. Retrieved from http://lcdtcorp.com/media/wysiwyg/descargas/5/16/30/kxtde100bx/Primeros_pasos_para_tarjetas_IP_virtuales.pdf

9. Velásquez, F. A. (2012). FACTIBILIDAD DE MIGRAR PBX CONVENCIONAL A $P B X-I P$. Retrieved from http://biblioteca.usac.edu.gt/tesis/08/08_0298_EO.pdf 